\title{
Hydrogen from wet air and sunlight in a tandem photoelectrochemical cell
}

\author{
K. Xu, ${ }^{1}$ A. Chatzitakis, ${ }^{1 *}$ E. Vøllestad, ${ }^{2}$ Q. Ruan, ${ }^{3}$ J. Tang, ${ }^{3}{ }^{T}$. Norby ${ }^{1}$ \\ ${ }^{1}$ Centre for Materials Science and Nanotechnology, Department of Chemistry, University of Oslo, FERMiO, \\ Gaustadalléen 21, NO-0349 Oslo, Norway \\ ${ }^{2}$ SINTEF Materials and Chemistry, POB 124 Blindern, NO-0314 Oslo, Norway \\ ${ }^{3}$ Department of Chemical Engineering, University College London, Torrington Place, London WC1E 7JE, \\ U.K. \\ *Corresponding author a.e.chatzitakis@smn.uio.no
}

\begin{abstract}
A solid-state photoelectrochemical cell (SSPEC) is an attractive approach for solar water splitting, especially when it comes to monolithic device design. In a SSPEC the electrodes distance is minimized, while the use of polymer-based membranes alleviates the need for liquid electrolytes, and at the same time they can separate the anode from the cathode. In this work, we have made and tested, firstly, a SSPEC cell with a Pt/C electrocatalyst as the cathode electrode, under purely gaseous conditions. The anode was supplied with air of $80 \%$ relative humidity $(\mathrm{RH})$ and the cathode with argon. Secondly, we replaced the Pt/C cathode with a photocathode consisting of $2 \mathrm{D}$ photocatalytic $\mathrm{g}-\mathrm{C}_{3} \mathrm{~N}_{4}$, which was placed in tandem with the photoanode (tandem-SSPEC). The tandem configuration showed a three-fold enhancement in the obtained photovoltage and a steady-state photocurrent density. The mechanism of operation is discussed in view of recent advances in surface proton conduction in absorbed water layers. The presented SSPEC cell is based on earth-abundant materials and provides a way towards systems of artificial photosynthesis, especially for areas where water sources are scarce and electrical grid
\end{abstract}


infrastructure is limited or nonexistent. The only requirements to make hydrogen are humidity and sunlight.

\section{Keywords}

solid-state photoelectrochemical cells; tandem photoelectrocatalysis; surface proton conduction; water vapor electrolysis; polymer electrolytes; earth-abundant materials 


\section{Introduction}

A fundamental societal concern is the impact of climate change on water quality and quantity [1]. It is also expected that the duration, magnitude and frequency of droughts on a worldwide basis will increase due to climate changes [2]. Water electrolysis is already an important and promising method for hydrogen generation and in the near future, clean water supplies will become a vital concern for this technology. Devices that can directly convert the solar energy to fuels, such as hydrogen, are highly desirable because the solar energy is absorbed and stored in the form of fuels in a single step [3-5]. Such "artificial photosynthesis" (AP) devices hold a great promise by mimicking nature in order to perform environmentally sustainable reactions, such as splitting water into $\mathrm{O}_{2}$ and $\mathrm{H}_{2}$, and $\mathrm{CO}_{2}$ fixation to sugars $[6,7]$. The device design is also very important and has an impact on the overall efficiency and cost [8]. Monolithic device designs, where the photoabsorbers are integrated together with the electrocatalysts, have certain advantages such as reduced area requirements, less frames and connections, as well as reduced footprint $[9,10]$.

In our previous work [11], we demonstrated a monolithic solid-state photoelectrochemical (SSPEC) water electrolysis cell, in which the photoanode and cathode electrodes were attached to opposite sides of a Nafion-based polymer electrolyte membrane. The role of the membrane is to separate the evolved gaseous products, support and minimize the distance between the electrodes (down to a few $\mu \mathrm{m}$ ) and provide protonic conductivity. The monolithic SSPEC cell was operated under asymmetric conditions i.e. $0.1 \mathrm{M} \mathrm{Na}_{2} \mathrm{SO}_{4}$ solution at the anode and gaseous Ar or air in the cathode compartment. There are a few more other works in the literature inspired by a polymer exchange membrane, PEM-like approach, where the photoanode is fed with a water stream $[12,13]$. Iwu et al. were the first to photoelectrolyze water vapor in an all-solid-state photoelectrochemical (PEC) cell [14], while Georgieva et al. have employed an all-solid-state PEC cell for the decomposition of organic vapors under UV and visible illumination [15]. 
Recently, the possibility of steam electrolysis in a high temperature PEC cell has been demonstrated by Brunauer and co-workers [16].

Inspired by recent advances in understanding of surface proton conduction in the adsorbed water layers in porous ceramic electrolytes $[17,18]$, we further developed our solid-state PEC cell (SSPEC), which operates now under ambient wet air conditions. The main advantage of this resource-efficient system is that the water source, which is the main reactant, is coming from the abundant and not exploited humidity in air or steam outputs from industry. This solid-state PEC (SSPEC) device is activated by sunlight and catalyzes the water splitting reaction in the gas phase, alleviating the need for clean water resources. Moreover, the noble-metal cathode, which is usually employed in PEC systems, is replaced by an earthabundant photocathode. We see that such a monolithic system is important for solar fuels generation in rural areas and in areas where grid infrastructure and water supply are limited or non-existent. The milder operating conditions (not requiring strong basic or acidic aqueous solutions) would decrease the degradation rate of the device components (electrodes, connections, gaskets etc.), a fact of great importance for a long-term performance [19].

In this work, we compare the PEC performance of two SSPEC configurations operated under gaseous conditions. The first one utilizes a Pt-based cathode (n-type | Nafion|C-Pt - SSPEC), as traditionally used in the vast majority of the PEC water splitting cells, while in the second, we replace the Pt cathode with a 2D photocathode $\mathrm{g}-\mathrm{C}_{3} \mathrm{~N}_{4}$ to make an n-type|Nafion/p-type tandem configuration (tandem SSPEC) and compare the two configurations. Finally, the mechanism for enabling water splitting is discussed in light of protonic surface diffusion on the photoanode, enabling larger electrochemically active area. It is worth mentioning that in both configurations the anode was supplied with wet air, while the cathode was supplied with dry Ar. 


\section{Experimental}

Experimental procedures and characterization methods are given in the supporting information (SI). Here, we describe the preparation of the SSPEC membranes and mounting cell, as well as the operating conditions.

As shown in Figure 1 the solid polymer electrolyte was prepared by drop-casting $200 \mu \mathrm{L}$ Nafion5 solution onto Cyclpr02 (Cyclopore track etched membrane of $0.2 \mu \mathrm{m}$ pores size, see SI for more information) and left to dry overnight. The Cyclpr5 (Cyclopore track etched membrane of $5 \mu \mathrm{m}$ pores size) was used in the tandem-SSPEC configuration as this membrane retained approx. $76 \%$ of its transparency after treatment with the Nafion5 (see Figure S1), while the Cyclpr02 became white. In both cases, the pores of the membranes were fully covered by the Nafion ${ }^{\circledR}$ solution (see Figure S2). The anode was adhered to the membrane by $20 \mu \mathrm{L}$ Nafion 20 solution as the binding agent, while the cathode was adhered to the opposite side by applying $10 \mu \mathrm{L}$ Nafion5 solution, and having the Pt-C or $\mathrm{g}-\mathrm{C}_{3} \mathrm{~N}_{4}$ side facing towards the membrane. Then, approx. $13 \mathrm{kPa}$ pressure was applied to the stack overnight in order to adhere the three components together and get a button-like PEC membrane. One may note that the Nafion ${ }^{\circledR}$ is at the same time the proton conducting polymer membrane and the binding agent for the fastening of the anode and cathode electrodes. At this point, we did not construct the $\mathrm{g}^{-} \mathrm{C}_{3} \mathrm{~N}_{4} \mid$ Nafion $\mid \mathrm{C}-\mathrm{Pt}$ configuration, which could assess the performance of the photocathode against the C-Pt as the anode electrode. For such a configuration, we need a better stabilization method for the photocathode and this will be addressed in our future works. 


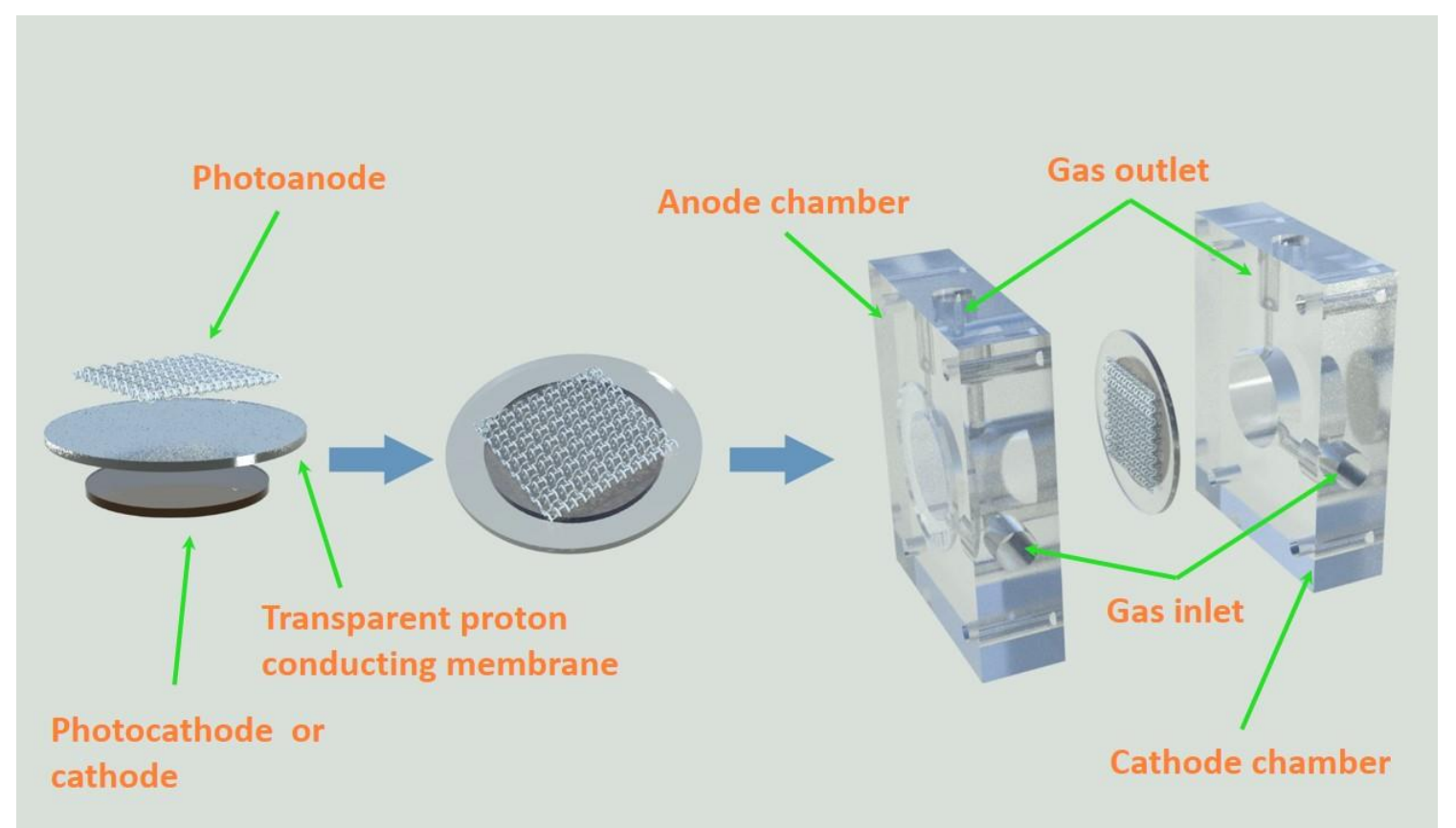

Figure 1: Schematic representation of the construction of the button-like PEC membrane, as well as its mounting on the custom-made solid-state PEC device. The PEC membrane is sandwiched between two silicone O-rings, which have appropriate copper wiring for connection to the potentiostat. The PEC device is of continuous flow type.

The nominal geometric area in the SSPEC configuration is considered as the nominal area of the $\mathrm{TiO}_{2}$ nanotubes (TNTs) mesh electrode, which was $1 \mathrm{~cm}^{2}$, while in the tandem-SSPEC configuration it is considered the area of the button cell, approx. $2 \mathrm{~cm}^{2}$, as in this case both electrodes contribute to the observed photocurrent. The anode compartment was supplied with air of $80 \%$ relative humidity (RH), while the cathode was supplied with dry Ar. The flow rates of both gases were set to $4.5 \mathrm{sccm}$ and the system was otherwise at ambient temperature $\left(25^{\circ} \mathrm{C}\right)$ and pressure $(1 \mathrm{~atm})$. Gas leakage and crossover were not determined, but assumed insignificant due to relatively small chemical gradients and absence of total pressure differences, and that the pores of the membrane were blocked by the Nafion ${ }^{\circledR}$ (see Figure 1 and Figure S2). 


\section{Results}

Scanning electron microscope (SEM) and X-ray diffraction (XRD) data are provided in Figures S3-S6. Herein, we focus on the photoelectrochemical characterization of the SSPEC cells, as well as the concept of the surface proton transport, which we suggest as the possible mechanism of the observed photoelectrochemical behaviour.

The open circuit voltage (OCV) response under dark and light conditions of the two SSPEC configurations is given in Figure 2a. It can be seen that the tandem-SSPEC cell produced an OCV of approx. 3 times higher than that produced by the SSPEC cell. This difference is attributed to the combined series photovoltages of the $\mathrm{n}$ - and $\mathrm{p}$ - semiconductors in the tandem-SSPEC cell, which increase the internal voltage of the device by a factor of three. The potential difference under light in both SSPEC cells is approx. $0.6 \mathrm{~V}$ (Figure 2a), which implies that the Fermi level $\left(E_{F}\right)$ in $g-C_{3} N_{4}$ reaches the $E_{F}$ of Pt. This suggests that proton reduction by $g-C_{3} N_{4}$ is thermodynamically favorable $[20,21]$. Moreover, the potential values in dark and light conditions for the SSPEC configuration are quite consistent, as they depend only on the time constants of charge separation and recombination of one photoelectrode, i.e. $\mathrm{TiO}_{2}$. In the tandem-SSPEC cell, it can be seen that these values differ between the first and the second light-ON, light-OFF cycle, but less in the third cycle. This behavior may be attributed to the combination of two photocatalysts, which have different time constants, as well as different photoelectrode microstructure and morphology.

(a)

(b) 


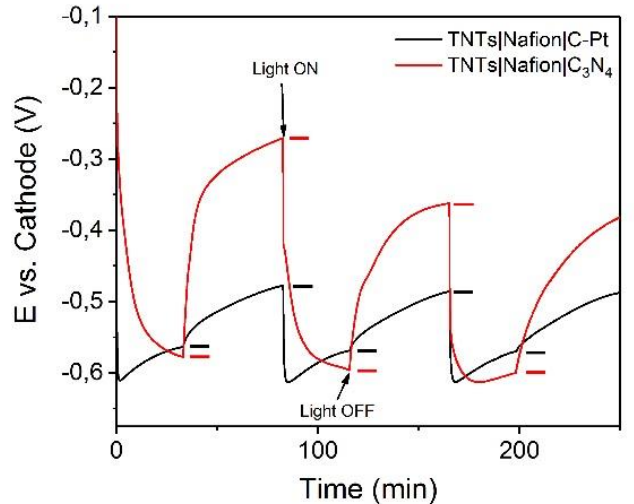

(c)

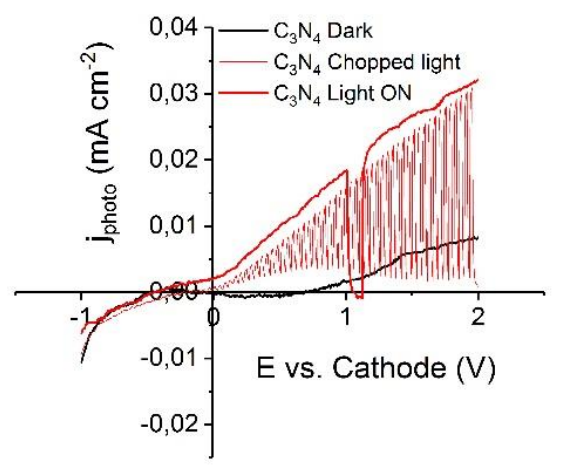

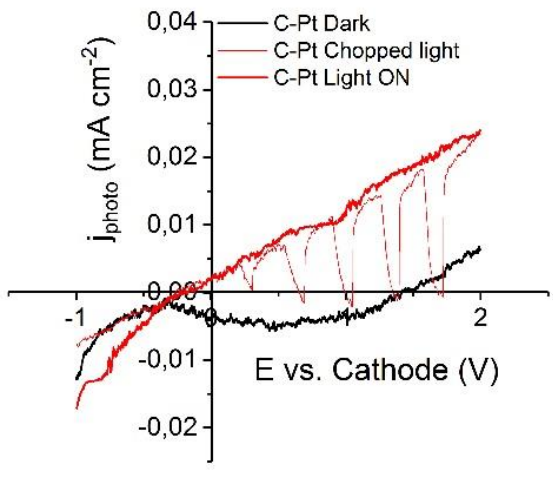

(d)

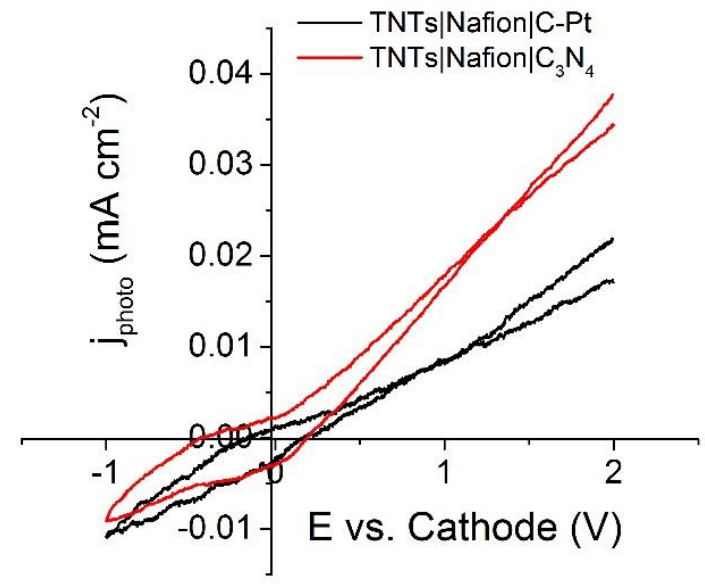


(e)

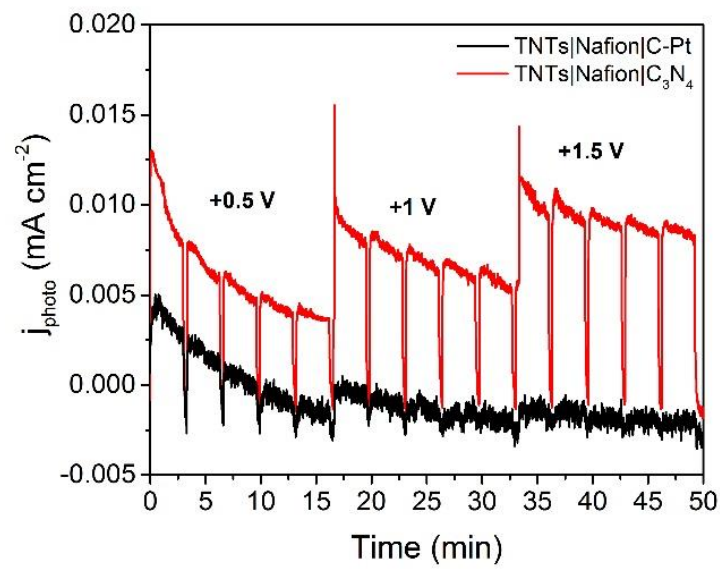

Figure 2: Open circuit voltage (OCV) of the two SSPEC configurations under dark (2000 s) and light conditions (1000 s) (a). Linear sweep voltammograms of the SSPEC (b) and tandem-SSPEC (c) cell configurations at a scan rate of $10 \mathrm{mV} \mathrm{s}^{-1}$ under chopped and continuous 1 Sun illumination. The fast response during the chopped light conditions is highlighted in the tandem-SSPEC cell by the much shorter light-ON, light-OFF steps. Cyclic voltammograms under 1 Sun illumination at a scan rate of 50 $\mathrm{mV} \mathrm{s}^{-1}(\mathrm{~d})$. Chronoamperometry at 3 different applied voltages. Light on period, $180 \mathrm{~s}$ and light off, 20 s (e).

The better PEC performance of the tandem-SSPEC cell is evident in the current-voltage curves in Figure $2 b, c$, and $d$ where the photocurrent density of the tandem-SSPEC cell exceeds the simple SSPEC. It should be noted that the observed photocurrent in the tandem configuration is normalized by a factor of 2 , while in the SSPEC configuration by a factor of 1 (see experimental). It should be noted that the dark scan and the dark from the chopped illumination curve do not match very well. A possible explanation might be that the scan rate used in the LSV experiments, was fast and the steady-state conditions were not reached. This may also explain why the current densities in the potentiostatic experiments of Figure 2e do not agree with the LSV values. A combination of appropriate scan rate and light-ON, light-OFF steps should be 
considered for such systems, where the water reactant is not in such an abundance as in a liquid PEC cell. Nevertheless, the tandem cell displays an improved steady-state photocurrent density, as depicted in the chronoamperometric curve (Fig.2e). It is also interesting to observe that the steady state photocurrent density in the case of the SSPEC cell reaches zero after a few minutes of operation at high applied potentials. Conversely, the tandem cell, displays a significant photocurrent density after 50 minutes of operation, with short light-OFF periods, which highlight the photocurrent generation. In general, we also observe that the photocurrent density is decreasing, but part of it is retrieved when light is turned on, after a short light-OFF period. This may be related to the heating of the surface of the photoanode, so that part of the physisorbed water layers is removed. The physisorbed water layers are compensated during the short light-OFF period. This hypothesis will be tested when the light-Off periods are increased and/or by thermostating the cell at higher temperatures, so that heating from the light is excluded. More details are given in the discussion section.

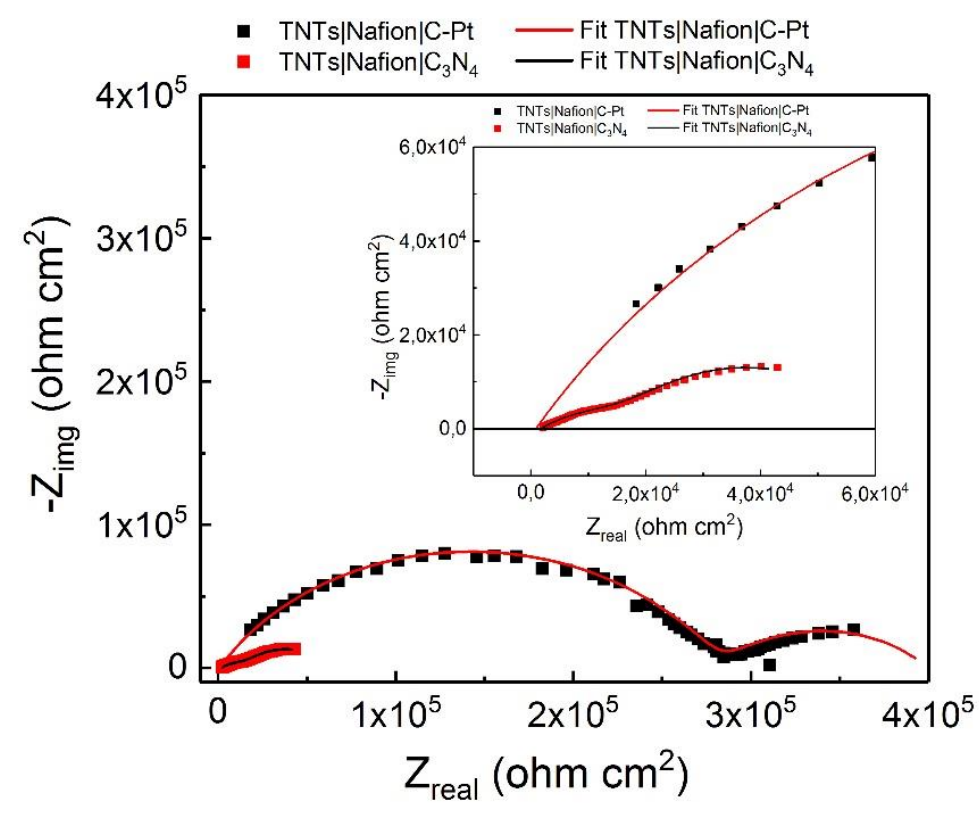

Figure 3: Nyquist plot of the two SSPEC cell configurations at $0.5 \mathrm{~V}$ vs. cathode and photocathode respectively, under 1 Sun illumination. Anode was provided with wet air $(80 \% \mathrm{RH})$ and the (photo)cathode with dry Ar. Inset: Fitted data in the high frequency domain. 
The superiority of the tandem configuration over the single SSPEC is also seen by the electrochemical impedance (EIS) measurements of Figure 3. The Nyquist plot is recorded at $0.5 \mathrm{~V}$ in a two-electrode configuration under 1 Sun illumination. Three depressed semicircles are observed in the tandem SSPEC cell, which are related to the charge transfer resistance over the two photoelectrodes, as well as a mass transfer resistance associated with chemical diffusion of reactants and products [17]. The charge transfer resistance of the SSPEC is clearly higher than the tandem-cell, and is attributed to the extra photovoltage provided by the photocathode, giving a higher electrochemical activity than the Pt cathode under illumination. In the dark though, a high to mid frequencies semicircle is seen for the SSPEC cell, but not for the tandem-SSPEC cell (see Figure S7a). The charge transfer semicircle in the tandem-SSPEC is most probably much higher in the dark and merged with the capacitive behavior in the low frequency domain, indicating a higher resistance for the photocathode in the dark, as compared to C-Pt. The equivalent circuits used to model the raw EIS data are given in Figure S7b, but details and the physical interpretation are beyond the scope of this communication. Deconvolution of the EIS spectra showed the same high frequency resistance (approx. $850 \mathrm{ohms} \mathrm{cm}^{2}$ in both dark and light conditions), which is related to the proton conductivity of the polymer electrolyte $\left(2.410^{-2} \mathrm{mS} \mathrm{cm}^{-1}\right)$ at room temperature and $80 \% \mathrm{RH}$. The overall resistance was found to be $27 \mathrm{kohms} \mathrm{cm}^{2}$ and $412 \mathrm{kohms} \mathrm{cm}^{2}$ for the tandem-SSPEC and SSPEC cell, respectively. This further supports the larger photocurrent density of the tandem cell.

\section{Discussion}

In this work we have demonstrated the generation of photovoltage and photocurrent of a solid-state PEC cell operating with humidified air as the reactant stream. Moreover, the steady state photocurrent density is significantly enhanced by replacing the Pt cathode with an earth-abundant photocathode, $g-\mathrm{C}_{3} \mathrm{~N}_{4}$ (Figure 
2d). The addition of the photocathode - due to its proper alignment with the photoanode (see Figure S8)

- adds to the generated photovoltage due to the series connection and enhances the proton reduction reaction at the cathode. In principle, the tandem-SSPEC resembles a z-scheme photocatalytic system [20], with the advantage that the oxidation and reduction products can be separated.

These SSPEC cells operate with humid gas as the supply of water, and thus resemble that of proton ceramic electrolyzers operated at intermediate to high temperatures. Contrary to traditional PEC cells, the charge transfer reaction (water oxidation) in such systems occur directly at the triple-phase-boundary (TPB) - the interface between electrolyte, electrode and gas, as the protons formed from the water oxidation reaction can directly "enter" the polymer electrolyte $[22,23]$. The mass transfer reactions adsorption, dissociation and diffusion - can occur along the entire electrode surface but is then limited by the chemical diffusion to the TPB for the charge transfer reaction to proceed. Thus, at low temperatures where the diffusivity is rather low, the total reaction is commonly limited to a small area close to the TPBs. For our current cell design, this would severely limit the electrochemically active area of the photoanode, and we would expect much higher electrode resistances than what is observed in our electrochemical characterizations.

However, recent advances in understanding transport properties of ceramics at low temperatures and humid atmospheres have revealed enhanced proton and water diffusivity along oxide surfaces due to the formation of adsorbed water layers, with a conductivity close to that of Nafion ${ }^{\circledR}$ at relative humidities above $60 \%$ [24-26]. This effect is particularly strong in hygroscopic materials such as $\mathrm{TiO}_{2}[27,28]$. Thus, it is reasonable to assume surface proton conduction is prevalent in our photoanodes operated at $80 \% \mathrm{RH}$, aiding the chemical diffusion of adsorbed species and reactants along the electrode surface and expanding the electrochemically active area beyond that of the triple-phase-boundaries. 
At the cathode, another TPB is formed between the gas phase, the electrolyte and the cathode and the protons are combined with the electrons and hydrogen gas is produced. The porosity of the cathode electrode, as well as the Ar flow ensures the $\mathrm{H}_{2}$ gas evolution and removal from the surface of the catalyst. On the other hand, the dry conditions in the cathode may have an impact on the water content close to the cathode side, resulting in the low proton conductivity observed in the present case $\left(2.410^{-2} \mathrm{mS} \mathrm{cm}^{-1}\right)$ and limited proton diffusivity along the surface. Adding humid Ar atmosphere could be a possibility, or a composite membrane, which stays hydrated at low $\mathrm{RH}[29,30]$. It should be mentioned that $\mathrm{H}_{2}$ was not detected due to the low current densities and the fact that the present system is of continuous flow rather than batch type, but further work is conducted in our group in order to minimize the series resistances, as well as optimize the polymer membrane performance and contact with the photoelectrodes. The choice, construction and placement of the photoelectrodes on the polymeric membrane is also another critical factor, which can open up several new paths for improvement. For example, the placement of "buried" photovoltaic (PV) junctions underneath the photoelectrodes can heavily improve the photocurrent densities for bias-free operation [31]. The room for improvement is also evident by the total series resistance measured for the two configurations, where by simply replacing the Pt-based cathode with a photocathode, the series resistance was decreased by an order of magnitude. 


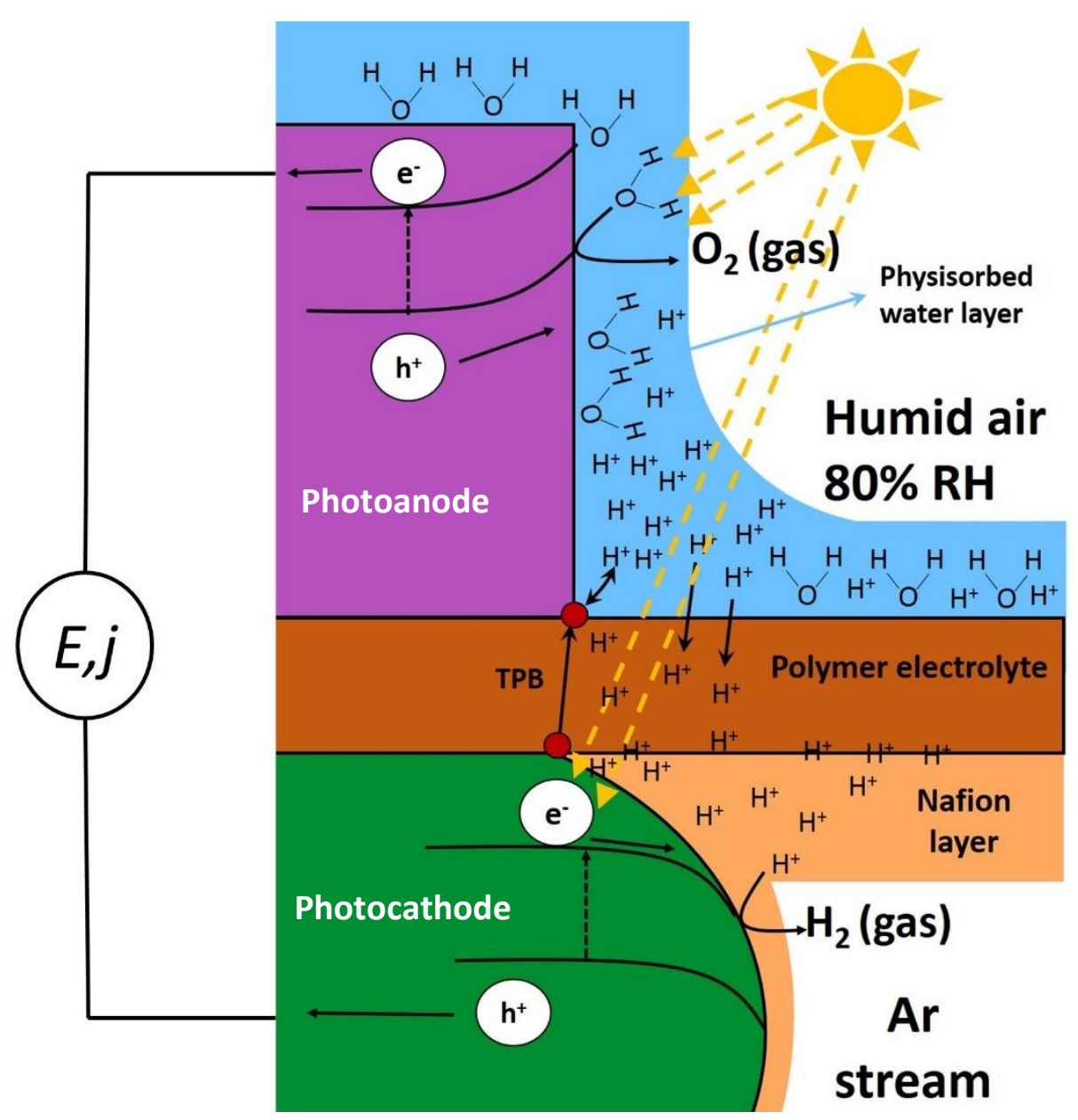

Figure 4: The concept of water vapor splitting and hydrogen production in a tandem-SSPEC cell. The surface water layer on the photoanode contributes to the proton transport towards the Nafion ${ }^{\circledR}$ membrane, while the lighter brown area in the photocathode represents the Nafion ${ }^{\circledR}$ layer, which apart from proton conduction, acts a binding agent. The photogenerated electrons in the photoanode are recombined in the external circuit with the photogenerated holes in the photocathode. In the case of the SSPEC cell, the photocathode is replaced by Pt nanoparticles, which are the catalysts for proton reduction and hydrogen evolution under the same conditions. The separating Nafion ${ }^{\circledR}$ membrane and the Nafion ${ }^{\circledR}$ layer are transparent. 


\section{Conclusions}

In this work we introduced the concept of water vapor splitting for hydrogen production in a solid-state PEC cell, after recent developments in the surface proton conduction in porous ceramic-based electrolytes. The traditionally Pt-based cathode is replaced by an earth-abundant photocathode, $g-C_{3} N_{4}$, which increased the intrinsic photo-induced electrical field by a factor of three. Moreover, we replaced the liquid electrolytes by a polymer proton conducting membrane based of Nafion ${ }^{\circledR}$. We envision that such a monolithic solid state PEC cell can play an important role for clean energy production in rural or other areas where grid infrastructure is limited or absent, and sources of clean water are scarce. What is needed is only sun and humid air.

\section{Acknowledgements}

Financial support from the Research Council of Norway (CO2BioPEC project 250261 and PH2BioCat project 239211) is acknowledged.

\section{References}

[1] Vörösmarty CJ, Green P, Salisbury J, Lammers RB. Global Water Resources: Vulnerability from Climate Change and Population Growth. Science. 2000;289:284.

[2] Nosrati K. Application of multivariate statistical analysis to incorporate physico-chemical surface water quality in low and high flow hydrology. Modeling Earth Systems and Environment. 2015;1:19.

[3] Tachibana Y, Vayssieres L, Durrant JR. Artificial photosynthesis for solar water-splitting. Nature Photonics. 2012;6:511.

[4] Joya KS, Joya YF, Ocakoglu K, van de Krol R. Water-Splitting Catalysis and Solar Fuel Devices: Artificial Leaves on the Move. Angewandte Chemie International Edition. 2013;52:10426-37. 
[5] Formal FL, Bourée WS, Prévot MS, Sivula K. Challenges towards Economic Fuel Generation from Renewable Electricity: The Need for Efficient Electro-Catalysis. CHIMIA International Journal for Chemistry. 2015;69:789-98.

[6] Musazade E, Voloshin R, Brady N, Mondal J, Atashova S, Zharmukhamedov SK, et al. Biohybrid solar cells: Fundamentals, progress, and challenges. Journal of Photochemistry and Photobiology C: Photochemistry Reviews. 2018;35:134-56.

[7] Voloshin RA, Bedbenov VS, Gabrielyan DA, Brady NG, Kreslavski VD, Zharmukhamedov SK, et al. Optimization and characterization of TiO2-based solar cell design using diverse plant pigments. Int J Hydrogen Energ. 2017;42:8576-85.

[8] Roger I, Shipman MA, Symes MD. Earth-abundant catalysts for electrochemical and photoelectrochemical water splitting. Nature Reviews Chemistry. 2017;1:0003.

[9] Lewis NS. Developing a scalable artificial photosynthesis technology through nanomaterials by design. Nature Nanotechnology. 2016;11:1010.

[10] Roel van de Krol, Grätzel M. Photoelectrochemical hydrogen production: Springer; 2012.

[11] Xu K, Chatzitakis A, Norby T. Solid-state photoelectrochemical cell with TiO2 nanotubes for water splitting. Photochemical \& Photobiological Sciences. 2017;16:10-6.

[12] Stoll T, Zafeiropoulos G, Dogan I, Genuit H, Lavrijsen R, Koopmans B, et al. Visible-light-promoted gasphase water splitting using porous WO3/BiVO4 photoanodes. Electrochemistry Communications. 2017;82:47-51.

[13] Zafeiropoulos G, Stoll T, Dogan I, Mamlouk M, van de Sanden MCM, Tsampas MN. Porous titania photoelectrodes built on a Ti-web of microfibers for polymeric electrolyte membrane photoelectrochemical (PEM-PEC) cell applications. Solar Energy Materials and Solar Cells. 2018;180:18495. 
[14] Iwu KO, Galeckas A, Kuznetsov AY, Norby T. Solid-state photoelectrochemical H2 generation with gaseous reactants. Electrochimica Acta. 2013;97:320-5.

[15] Georgieva J, Armyanov S, Poulios I, Sotiropoulos S. An all-solid photoelectrochemical cell for the photooxidation of organic vapours under ultraviolet and visible light illumination. Electrochemistry Communications. 2009;11:1643-6.

[16] Brunauer GC, Rotter B, Walch G, Esmaeili E, Opitz AK, Ponweiser K, et al. UV-Light-Driven Oxygen Pumping in a High-Temperature Solid Oxide Photoelectrochemical Cell. Advanced Functional Materials. 2016;26:120-8.

[17] Stub $S \varnothing$, Vøllestad E, Norby T. Mechanisms of Protonic Surface Transport in Porous Oxides: Example of YSZ. The Journal of Physical Chemistry C. 2017;121:12817-25.

[18] Scherrer B, Schlupp Meike VF, Stender D, Martynczuk J, Grolig Jan G, Ma H, et al. Thin Films: On Proton Conductivity in Porous and Dense Yttria Stabilized Zirconia at Low Temperature (Adv. Funct. Mater. 15/2013). Advanced Functional Materials. 2013;23:1858-.

[19] Nocera DG. The Artificial Leaf. Accounts of Chemical Research. 2012;45:767-76.

[20] Wang Y, Suzuki H, Xie J, Tomita O, Martin DJ, Higashi M, et al. Mimicking Natural Photosynthesis: Solar to Renewable H2 Fuel Synthesis by Z-Scheme Water Splitting Systems. Chemical Reviews. 2018;118:5201-41.

[21] Martin DJ, Reardon PJT, Moniz SJA, Tang J. Visible Light-Driven Pure Water Splitting by a NatureInspired Organic Semiconductor-Based System. Journal of the American Chemical Society. 2014;136:12568-71.

[22] Kreuer KD. Proton-Conducting Oxides. Annual Review of Materials Research. 2003;33:333-59.

[23] Kreuer K-D. Proton Conductivity: Materials and Applications. Chemistry of Materials. 1996;8:610-41. 
[24] Kim S, Avila-Paredes HJ, Wang S, Chen C-T, De Souza RA, Martin M, et al. On the conduction pathway for protons in nanocrystalline yttria-stabilized zirconia. Physical Chemistry Chemical Physics. 2009;11:3035-8.

[25] Stub SO, Vollestad E, Norby T. Protonic surface conduction controlled by space charge of intersecting grain boundaries in porous ceramics. Journal of Materials Chemistry A. 2018;6:8265-70.

[26] T. CM. Nanoporous Anatase Thin Films as Fast Proton-Conducting Materials. Advanced Materials. 2006;18:371-4.

[27] Di Vona ML, Ahmed Z, Bellitto S, Lenci A, Traversa E, Licoccia S. SPEEK-TiO2 nanocomposite hybrid proton conductive membranes via in situ mixed sol-gel process. Journal of Membrane Science. 2007;296:156-61.

[28] Stub S $\varnothing$, Thorshaug K, Rørvik PM, Norby T, Vøllestad E. The influence of acceptor and donor doping on the protonic surface conduction of TiO2. Physical Chemistry Chemical Physics. 2018;20:15653-60.

[29] Yang J, Shen PK, Varcoe J, Wei Z. Nafion/polyaniline composite membranes specifically designed to allow proton exchange membrane fuel cells operation at low humidity. Journal of Power Sources. 2009;189:1016-9.

[30] Shao Z-G, Joghee P, Hsing IM. Preparation and characterization of hybrid Nafion-silica membrane doped with phosphotungstic acid for high temperature operation of proton exchange membrane fuel cells. Journal of Membrane Science. 2004;229:43-51.

[31] Verlage E, Hu S, Liu R, Jones RJR, Sun K, Xiang C, et al. A monolithically integrated, intrinsically safe, $10 \%$ efficient, solar-driven water-splitting system based on active, stable earth-abundant electrocatalysts in conjunction with tandem III-V light absorbers protected by amorphous TiO2 films. Energy \& Environmental Science. 2015;8:3166-72. 